\title{
THREE NONNEGATIVE SOLUTIONS FOR SECOND-ORDER IMPULSIVE DIFFERENTIAL EQUATIONS WITH A THREE-POINT BOUNDARY VALUE PROBLEM
}

\author{
JIANLI LI ${ }^{\otimes 1}$ and JIANHUA SHEN ${ }^{2}$
}

(Received 9 September, 2006; revised 4 January, 2008)

\begin{abstract}
In this paper, by using the Leggett-Williams fixed point theorem, we prove the existence of three nonnegative solutions to second-order nonlinear impulsive differential equations with a three-point boundary value problem.
\end{abstract}

2000 Mathematics subject classification: 34B10, 34B37.

Keywords and phrases: differential equation, boundary value problem, nonnegative solution, fixed point theorem.

\section{Introduction}

Let $0=t_{0}<t_{1}<\cdots<t_{m}<t_{m+1}=1$ be given. In this paper we present results which guarantee the existence of three nonnegative solutions to the second-order impulsive equation

$$
\left\{\begin{aligned}
y^{\prime \prime}(t)+h(t) f(y(t))=0 & \text { for } t \in(0,1) \backslash\left\{t_{1}, \ldots, t_{m}\right\} \\
\Delta y\left(t_{k}\right)=I_{k}\left(y\left(t_{k}^{-}\right)\right), & k=1, \ldots, m \\
\Delta y^{\prime}\left(t_{k}\right)=J_{k}\left(y\left(t_{k}^{-}\right)\right), & k=1, \ldots, m \\
y(0)=0 & \\
\alpha y(\eta)=y(1) &
\end{aligned}\right.
$$

where $0<\eta<1, \quad 0<\alpha<1 / \eta, \quad \Delta y\left(t_{k}\right)=y\left(t_{k}^{+}\right)-y\left(t_{k}^{-}\right)$, and $y\left(t_{k}^{+}\right)$and $y\left(t_{k}^{-}\right)$ respectively denote the right limit and left limit of $y(t)$ at $t=t_{k}$. Also $\Delta y^{\prime}\left(t_{k}\right)=$ $y^{\prime}\left(t_{k}^{+}\right)-y^{\prime}\left(t_{k}^{-}\right)$. We define the Banach space ( $r=0$ or 2 in this paper),

\footnotetext{
${ }^{1}$ Department of Mathematics, Hunan Normal University, Changsha, Hunan 410081, China; e-mail: 1jianli@sina.com.

${ }^{2}$ Department of Mathematics, College of Huaihua, Huaihua, Hunan 418008, China.

(C) Australian Mathematical Society 2008, Serial-fee code 0334-2700/08
} 


$$
\begin{gathered}
P C^{r}[0,1]=\left\{y:[0,1] \times R: \text { for all } j=0, \ldots, m \text { there exists } y_{j} \in C^{r}\left[t_{j}, t_{j+1}\right]\right. \\
\text { such that } \left.y=y_{j} \text { on }\left(t_{j}, t_{j+1}\right], y(0)=y_{0}(0)\right\}
\end{gathered}
$$

with the norm

$$
\|y\|_{P C^{r}}=\max \left\{\|y\|,\left\|y^{\prime}\right\|, \ldots,\left\|y^{(r)}\right\|\right\}
$$

Here

$$
\|y\|=\sup \{|y(t)|, t \in[0,1]\} .
$$

By a solution to (1.1) we mean a function $y \in P C^{2}[0,1]$ which satisfies (1.1). In (1.1), as $\alpha=0$, the existence of three nonnegative solutions was considered by Agarwal and O'Regan [3], and as $I_{k}\left(y\left(t_{k}^{-}\right)\right)=J_{k}\left(y\left(t_{k}^{-}\right)\right) \equiv 0, k=1, \ldots, m$, the positive solution was obtained by Ma [6]. In this paper, motivated by [3] and [6], we shall show the existence of three nonnegative solutions to (1.1) by the LeggettWilliams fixed point theorem [5]. Recently [1-4, 7, 8] this fixed point theorem has been used to establish multiplicity results for differential, integral and difference equations.

Now we present some preliminaries which will be needed in Section 3. First, $E=(E,\|\cdot\|)$ is a Banach space and $P \subset E$ is a cone. By a concave nonnegative continuous functional $\psi$ on $P$ we mean a continuous mapping $\psi: P \rightarrow[0, \infty)$ with

$$
\psi(\lambda x+(1-\lambda) y) \geq \lambda \psi(x)+(1-\lambda) \psi(y) \text { for all } x, y \in P \text { and all } \lambda \in[0,1] .
$$

Let $K, L, r>0$ be constants with $P$ and $\psi$ as defined above. Let

$$
P_{K}=\{y \in P:\|y\|<K\} \quad \text { and } \quad P(\psi, r, L)=\{y \in P: \psi(y) \geq r \text { and }\|y\| \leq L\} .
$$

We now state the Leggett-Williams fixed point theorem [5].

Theorem 1.1. Let $E=(E,\|\cdot\|)$ be a Banach space, $P \subset E$ a cone of $E$ and $R>0$ a constant. Suppose there exists a concave nonnegative continuous functional $\psi$ on $P$ with $\psi(y) \leq\|y\|$ for all $y \in \bar{P}_{R}$ and let $A: \bar{P}_{R} \rightarrow \bar{P}_{R}$ be a continuous compact map. Assume there are numbers $r, L$ and $K$ with $0<r<L<K \leq R$ such that:

(H1) $\{y \in P(\psi, L, K): \psi(y)>L\} \neq \emptyset$ and $\psi(A y)>L$ for all $y \in P(\psi, L, K)$;

(H2) $\|A y\|<r$ for all $y \in \bar{P}_{r}$; and

(H3) $\psi(A y)>L$ for all $y \in P(\psi, L, R)$ with $\|A y\|>K$.

Then $A$ has at least three fixed points $y_{1}, y_{2}$ and $y_{3}$ in $\bar{P}_{R}$. Furthermore

$y_{1} \in P_{r}, \quad y_{2} \in\{y \in P(\psi, L, R): \psi(y)>L\} \quad$ and $\quad y_{3} \in \bar{P}_{R} \backslash\left(P(\psi, L, R) \cup \bar{P}_{r}\right)$.

\section{Some lemmas}

Consider the impulsive integral equation

$$
y(t)=\int_{0}^{1} H(t, s) h(s) f(y(s)) d s+\sum_{k=1}^{m} W_{k}(t, y), \quad t \in[0,1],
$$


where

$$
\begin{gathered}
H(t, s)=\frac{1}{1-\alpha \eta} t(1-s)-U(t, s)-\frac{\alpha}{1-\alpha \eta} V(t, s), \quad 0 \leq t, s \leq 1, \\
U(t, s)=\left\{\begin{array}{ll}
t-s, & s \leq t, \\
0, & t \leq s,
\end{array} \quad V(t, s)= \begin{cases}t(\eta-s), & s \leq \eta, \\
0, & \eta \leq s,\end{cases} \right.
\end{gathered}
$$

and for $k=1, \ldots, m$

$$
W_{k}(t, y)=\left\{\begin{array}{lc}
\frac{1-t-\alpha \eta+\alpha t}{1-\alpha \eta}\left[I_{k}\left(y\left(t_{k}^{-}\right)\right)-t_{k} J_{k}\left(y\left(t_{k}^{-}\right)\right)\right], & 0<t_{k}<\min \{t, \eta\}, \\
\frac{t}{1-\alpha \eta}\left\{-I_{k}(y)-\left(1-t_{k}\right) J_{k}(y)+\alpha\left[I_{k}(y)+\left(\eta-t_{k}\right) J_{k}(y)\right]\right\}, & t \leq t_{k}<\max \{t, \eta\}, \\
\frac{1}{1-\alpha \eta}\left\{(1-\alpha \eta)\left(I_{k}(y)-t_{k} J_{k}(y)\right)-t\left[I_{k}(y)-\left(t_{k}-\alpha \eta\right) J_{k}(y)\right]\right\}, & \eta \leq t_{k}<\max \{t, \eta\}, \\
\frac{t}{1-\alpha \eta}\left[-I_{k}\left(y\left(t_{k}^{-}\right)\right)-\left(1-t_{k}\right) J_{k}\left(y\left(t_{k}^{-}\right)\right)\right], & \max \{t, \eta\} \leq t_{k}<1 .
\end{array}\right.
$$

LEMMA 2.1. We have that $y \in P C[0,1] \cap P C^{2}[0,1]$ is a solution of $(1.1)$ if and only if $y \in P C[0,1]$ is a solution of the integral equation (2.1).

PROOF. Suppose that $y \in P C[0,1]$ is a solution of (2.1). Then for $t \neq t_{k}$,

$$
\begin{aligned}
y^{\prime}(t)= & \frac{1}{1-\alpha \eta} \int_{0}^{1}(1-s) h(s) f(y(s)) d s-\frac{\alpha}{1-\alpha \eta} \int_{0}^{\eta}(\eta-s) h(s) f(y(s)) d s \\
& -\frac{1}{1-\alpha \eta} \sum_{0<t_{k}<1}\left[J_{k}(y)\left(1-t_{k}\right)+I_{k}(y)\right] \\
& +\frac{\alpha}{1-\alpha \eta} \sum_{0<t_{k}<\eta}\left[J_{k}(y)\left(\eta-t_{k}\right)+I_{k}(y)\right] \\
& -\int_{0}^{t} h(s) f(y(s)) d s+\sum_{0<t_{k}<t} J_{k}(y), \\
y^{\prime \prime}(t)= & -h(t) f(y(t)),
\end{aligned}
$$

and for $t=t_{k}$,

$$
\begin{gathered}
\Delta y\left(t_{k}\right)=y\left(t_{k}^{+}\right)-y\left(t_{k}^{-}\right)=I_{k}\left(y\left(t_{k}^{-}\right)\right), \\
\Delta y^{\prime}\left(t_{k}\right)=y^{\prime}\left(t_{k}^{+}\right)-y^{\prime}\left(t_{k}^{-}\right)=J_{k}\left(y\left(t_{k}^{-}\right)\right),
\end{gathered}
$$

and

$$
y(0)=0, \quad \alpha y(\eta)=y(1) .
$$

So $y$ is a solution of (1.1). 
On the other hand, if $y$ is a solution of (2.1), then

$$
\begin{gathered}
y^{\prime}(t)=y^{\prime}(0)-\int_{0}^{t} h(s) f(y(s)) d s+\sum_{0<t_{k}<t} J_{k}(y), \\
y(t)=y^{\prime}(0) t-\int_{0}^{t}(t-s) h(s) f(y(s)) d s+\sum_{0<t_{k}<t}\left[J_{k}(y)\left(t-t_{k}\right)+I_{k}(y)\right] .
\end{gathered}
$$

This and the boundary value condition $y(0)=0$ and $\alpha y(\eta)=y(1)$ imply that

$$
\begin{aligned}
y^{\prime}(0)= & \frac{1}{1-\alpha \eta} \int_{0}^{1}(1-s) h(s) f(y(s)) d s-\frac{\alpha}{1-\alpha \eta} \int_{0}^{\eta}(\eta-s) h(s) f(y(s)) d s \\
& -\frac{1}{1-\alpha \eta} \sum_{0<t_{k}<1}\left[J_{k}(y)\left(1-t_{k}\right)+I_{k}(y)\right] \\
& +\frac{\alpha}{1-\alpha \eta} \sum_{0<t_{k}<\eta}\left[J_{k}(y)\left(\eta-t_{k}\right)+I_{k}(y)\right] .
\end{aligned}
$$

Therefore

$$
\begin{aligned}
y(t)= & \frac{t}{1-\alpha \eta} \int_{0}^{1}(1-s) h(s) f(y(s)) d s-\frac{\alpha t}{1-\alpha \eta} \int_{0}^{\eta}(\eta-s) h(s) f(y(s)) d s \\
& -\frac{t}{1-\alpha \eta} \sum_{0<t_{k}<1}\left[J_{k}(y)\left(1-t_{k}\right)+I_{k}(y)\right] \\
& +\frac{\alpha t}{1-\alpha \eta} \sum_{0<t_{k}<\eta}\left[J_{k}(y)\left(\eta-t_{k}\right)+I_{k}(y)\right] \\
& -\int_{0}^{t}(t-s) h(s) f(y(s)) d s+\sum_{0<t_{k}<t}\left[J_{k}(y)\left(t-t_{k}\right)+I_{k}(y)\right] .
\end{aligned}
$$

For $0 \leq t \leq \eta$

$$
\begin{aligned}
y(t)= & \int_{0}^{1} H(t, s) h(s) f(y(s)) d s+\frac{1-t-\alpha \eta+\alpha t}{1-\alpha \eta} \sum_{0<t_{k}<t}\left[I_{k}(y)-t_{k} J_{k}(y)\right] \\
& +\frac{t}{1-\alpha \eta} \sum_{t \leq t_{k}<\eta}\left\{-I_{k}(y)-\left(1-t_{k}\right) J_{k}(y)+\alpha\left[I_{k}(y)+\left(\eta-t_{k}\right) J_{k}(y)\right]\right\} \\
& +\frac{t}{1-\alpha \eta} \sum_{\eta \leq t_{k}<1}\left[-I_{k}(y)-\left(1-t_{k}\right) J_{k}(y)\right] .
\end{aligned}
$$


For $\eta \leq t \leq 1$,

$$
\begin{aligned}
y(t)= & \int_{0}^{1} H(t, s) h(s) f(y(s)) d s+\frac{1-t-\alpha \eta+\alpha t}{1-\alpha \eta} \sum_{0<t_{k}<\eta}\left[I_{k}(y)-t_{k} J_{k}(y)\right] \\
& +\frac{1}{1-\alpha \eta} \sum_{\eta \leq t_{k}<t}\left\{(1-\alpha \eta)\left(I_{k}(y)-t_{k} J_{k}(y)\right)-t\left[I_{k}(y)-\left(t_{k}-\alpha \eta\right) J_{k}(y)\right]\right\} \\
& +\frac{t}{1-\alpha \eta} \sum_{\eta \leq t_{k}<1}\left[-I_{k}(y)-\left(1-t_{k}\right) J_{k}(y)\right] .
\end{aligned}
$$

So

$$
y(t)=\int_{0}^{1} H(t, s) h(s) f(y(s)) d s+\sum_{k=1}^{m} W_{k}(t, y), \quad t \in[0,1] .
$$

LEMMA 2.2. We have that:

(1) $H:[0,1] \times[0,1] \rightarrow[0,+\infty)$ is continuous; and

(2) $H(t, s) \leq M_{1} H(s, s)$ for all $t, s \in[0,1]$,

$H(t, s) \geq M_{2} H(s, s)$ for $s \in[0,1], t \in\left[a_{k}, b_{k}\right]$,

where

$$
\begin{aligned}
& M_{1}=\max \left\{\frac{1-\alpha \eta}{\alpha(1-\eta)}, \frac{1+\alpha \eta}{\eta}\right\}, \\
& M_{2}=\min \left\{\frac{t_{1}}{4}, \frac{(1-\alpha \eta)\left(1-t_{m}\right)}{4}, \frac{(1-\alpha \eta)\left(1-t_{m}\right)}{4 \alpha(1-\eta)}\right\}, \\
& a_{k}=\frac{3 t_{k}+t_{k+1}}{4}, \quad b_{k}=\frac{t_{k}+3 t_{k+1}}{4} \quad \text { for } k \in\{0,1, \ldots, m\} .
\end{aligned}
$$

PROOF. Part (1) is part (1) of [9, Lemma 3.1]. Now we prove part (2). We divide the proof into the following six cases.

(i) If $0 \leq s \leq t \leq \eta$, then

$$
\begin{aligned}
& \frac{H(t, s)}{H(s, s)}=\frac{1-\alpha \eta+t(\alpha-1)}{1-\alpha \eta+s(\alpha-1)} \leq \begin{cases}1, & \alpha \geq 1, \\
\frac{1-\alpha \eta}{\alpha(1-\eta)}, & \alpha<1, \\
\frac{H(t, s)}{H(s, s)}=\frac{1-\alpha \eta+t(\alpha-1)}{1-\alpha \eta+s(\alpha-1)} \geq(1-\alpha \eta)(1-t) \geq(1-\alpha \eta) \frac{1-t_{m}}{4}, \\
\quad t \in\left[a_{k}, b_{k}\right] .\end{cases}
\end{aligned}
$$

(ii) If $0 \leq t \leq s \leq \eta$, then

$$
\begin{aligned}
& \frac{H(t, s)}{H(s, s)}=\frac{t}{s} \leq 1, \\
& \frac{H(t, s)}{H(s, s)}=\frac{t}{s} \geq \frac{t_{1}}{4}, \quad t \in\left[a_{k}, b_{k}\right] .
\end{aligned}
$$


(iii) If $0 \leq s \leq \eta \leq t \leq 1$, then

$$
\begin{aligned}
& \frac{H(t, s)}{H(s, s)}=\frac{1-\alpha \eta+t(\alpha-1)}{1-\alpha \eta+s(\alpha-1)} \leq \begin{cases}1, & \alpha \geq 1, \\
\frac{1-\alpha \eta}{\alpha(1-\eta)}, & \alpha<1,\end{cases} \\
& \frac{H(t, s)}{H(s, s)} \geq \begin{cases}1-t \geq \frac{1-t_{m}}{4}, & \alpha \leq 1, t \in\left[a_{k}, b_{k}\right], \\
\frac{(1-\alpha \eta)(1-t)}{\alpha(1-\eta)} \geq \frac{(1-\alpha \eta)\left(1-t_{m}\right)}{4 \alpha(1-\eta)}, & \alpha>1, t \in\left[a_{k}, b_{k}\right] .\end{cases}
\end{aligned}
$$

(iv) If $0 \leq t \leq \eta \leq s \leq 1$, then

$$
\begin{aligned}
& \frac{H(t, s)}{H(s, s)}=\frac{t}{s} \leq 1, \\
& \frac{H(t, s)}{H(s, s)}=\frac{t}{s} \geq \frac{t_{1}}{4}, \quad t \in\left[a_{k}, b_{k}\right] .
\end{aligned}
$$

(v) If $\eta \leq s \leq t \leq 1$, then

$$
\begin{aligned}
& \frac{H(t, s)}{H(s, s)}=\frac{s(1-t)+\alpha \eta(t-s)}{s(1-s)} \leq \frac{s(1-s)+\alpha \eta(1-s)}{s(1-s)}=\frac{s+\alpha \eta}{s} \leq \frac{1+\alpha \eta}{\eta}, \\
& \frac{H(t, s)}{H(s, s)}=\frac{s(1-t)+\alpha \eta(t-s)}{s(1-s)} \geq \frac{1-t}{1-s} \geq \frac{1}{1-\eta} \frac{1-t_{m}}{4}, \quad t \in\left[a_{k}, b_{k}\right] .
\end{aligned}
$$

(vi) If $\eta \leq t \leq s \leq 1$, then

$$
\begin{aligned}
& \frac{H(t, s)}{H(s, s)}=\frac{t}{s} \leq 1, \\
& \frac{H(t, s)}{H(s, s)}=\frac{t}{s} \geq \frac{t_{1}}{4}, \quad t \in\left[a_{k}, b_{k}\right] .
\end{aligned}
$$

Thus

$$
\begin{array}{ll}
H(t, s) \leq M_{1} H(s, s) & \text { for } t, s \in[0,1], \\
H(t, s) \geq M_{2} H(s, s) & \text { for } s \in[0,1], t \in\left[a_{k}, b_{k}\right] .
\end{array}
$$

REMARK 2.1. Note that $M_{1}>1$.

\section{Existence}

We will use Theorem 1.1 to establish the existence of three nonnegative solutions to (1.1). The following conditions will be assumed: 


$$
\begin{aligned}
& h \in C(0,1) \text { with } h>0 \text { on }(0,1) \text { and } h \in L^{1}[0,1] \text {, } \\
& f:[0, \infty) \rightarrow[0, \infty) \text { is continuous and nondecreasing, } \\
& I_{k}, J_{k}:[0, \infty) \rightarrow R \text { are continuous for } k=1, \ldots, m, \\
& t_{k} J_{k}(v) \leq I_{k}(v) \leq\left(t_{k}-1\right) J_{k}(v) \text { for } v \geq 0 \text { and } k=1, \ldots, m, \\
& \begin{cases}I_{k}(v) \geq\left(t_{k}-\eta\right) J_{k}(v) & \text { for } v \geq 0, t_{k}<\eta \text { and } k \in\{1, \ldots, m\}, \\
I_{k}(v) \leq\left(t_{k}-\alpha \eta\right) J_{k}(v) & \text { for } v \geq 0, t_{k} \geq \eta \text { and } k \in\{1, \ldots, m\},\end{cases} \\
& \left\{\begin{array}{l}
W_{k}(t, u) \leq \Omega_{k}\left(u\left(t_{k}\right)\right) \text { for } t \in[0,1] \text { and } u \in C[0,1] \text { with } u \geq 0, \\
\text { and with } \Omega_{k} \geq 0 \text { continuous and nondecreasing on }[0, \infty),
\end{array}\right. \\
& \exists r>0 \text { with } f(r) \sup _{t \in[0,1]} \int_{0}^{1} H(t, s) h(s) d s+\sum_{i=1}^{m} \Omega_{i}(r)<r, \\
& \exists L>r \text { with } f(L) \min _{k \in\{0,1, \ldots, m\}} \min _{t \in\left[a_{k}, b_{k}\right]} \int_{a_{k}}^{b_{k}} H(t, s) h(s) d s>L,
\end{aligned}
$$

and

$$
\exists R \geq L M^{-1} M_{1} \text { with } f(R) \sup _{t \in[0,1]} \int_{0}^{1} H(t, s) h(s) d s+\sum_{j=1}^{m} \Omega_{j}(R) \leq R,
$$

where

$$
M=\min \left\{c_{0}, M_{2}\right\}
$$

THEOREM 3.1. Suppose that (3.1)-(3.10) hold. Then (1.1) has at least three nonnegative solutions $y_{1}, y_{2}$ and $y_{3}$ in $P C^{2}[0,1]$ such that

$$
\left\|y_{1}\right\|<r, \quad y_{2}(t)>L \quad \text { for } t \in\left[a_{k}, b_{k}\right], k \in\{0,1, \ldots, m\},
$$

and

$$
\left\|y_{3}\right\|>r \quad \text { with } \min _{k \in\{0, \ldots, m\}} \min _{t \in\left[a_{k}, b_{k}\right]} y_{3}(t)<L
$$

PROOF. Let

$$
E=(P C[0,1],\|\cdot\|) \quad \text { and } \quad P=\{u \in P C[0,1], u(t) \geq 0 \text { for } t \in[0,1]\} .
$$

Now let $A: P C[0,1] \rightarrow P C[0,1]$ be defined by

$$
A y(t)=\int_{0}^{1} H(t, s) h(s) f(y(s)) d s+\sum_{k=1}^{m} W_{k}(t, y) \quad \text { for } t \in[0,1] .
$$

For $y \geq 0$ the conditions (3.1), (3.2), (3.4) and (3.5) imply that $A y(t) \geq 0$ for $t \in[0,1]$. So $A(P) \subset P$. It is easy to show that $A: P \rightarrow P$ is continuous and completely continuous [3]. 
For $y \in P$, let

$$
\psi(y)=\min _{k \in\{0,1, \ldots, m\}} \min _{t \in\left[a_{k}, b_{k}\right]} y(t) .
$$

Then $\psi$ is a nonnegative continuous concave functional on $P$ with $\psi(y) \leq\|y\|$ for $y \in P$. Next choose and fix $K$ so that

$$
L M_{1} M^{-1} \leq K \leq R
$$

First, we prove that condition (H2) of Theorem 1.1 holds. To do this, let $y \in \bar{P}_{r}$, then $0 \leq y \leq r$. Conditions (3.2), (3.6) and (3.7) imply for $t \in[0,1]$ that

$$
A y(t) \leq f(r) \sup _{t \in[0,1]} \int_{0}^{1} H(t, s) h(s) d s+\sum_{k=1}^{m} \Omega_{k}(r)<r .
$$

So

$$
\|A y\|<r .
$$

This shows that condition (H2) of Theorem 1.1 follows. Also $A: \bar{P}_{R} \rightarrow \bar{P}_{R}$ since, if $y \in \bar{P}_{R}$, then

$$
\|A y\| \leq f(R) \sup _{t \in[0,1]} \int_{0}^{1} H(t, s) h(s)+\sum_{k=1}^{m} \Omega_{k}(R) \leq R .
$$

Next, we show that $\{y \in P(\psi, L, K): \psi(y)>L\} \neq \emptyset$ and $\psi(A y)>L$ for all $y \in P(\psi, L, K)$. In fact, take $u(t) \equiv(L+K) / 2$ for $t \in[0,1]$, then

$$
u \in\{y \in P(\psi, L, K): \psi(y)>L\} .
$$

Moreover, for $y \in P(\psi, L, K)$, then $\psi(y)=\min _{k \in\{0,1, \ldots, m\}} \min _{t \in\left[a_{k}, b_{k}\right]} y(t) \geq L$ and $\|y\| \leq K$, so for each $k \in\{0,1, \ldots, m\}$, we have

$$
y(t) \in[L, K] \text { for } t \in\left[a_{k}, b_{k}\right] .
$$

This together with (3.8) yields

$$
\begin{aligned}
\psi(A y) & =\min _{k \in\{0,1, \ldots, m\}} \min _{t \in\left[a_{k}, b_{k}\right]}\left(\int_{0}^{1} H(t, s) h(s) f(y(s)) d s+\sum_{j=1}^{m} W_{j}(t, y)\right) \\
& \geq \min _{k \in\{0,1, \ldots, m\}} \min _{t \in\left[a_{k}, b_{k}\right]} \int_{a_{k}}^{b_{k}} H(t, s) h(s) f(y(s)) d s \\
& \geq f(L) \min _{k \in\{0,1, \ldots, m\}} \min _{t \in\left[a_{k}, b_{k}\right]} \int_{a_{k}}^{b_{k}} H(t, s) h(s) d s>L .
\end{aligned}
$$

So condition (H1) of Theorem 1.1 is satisfied. 
Finally, we assert that if $y \in P(\psi, L, R)$ and $\|A y\|>K$, then $\psi(A y)>L$. To see this, let $y \in P(\psi, L, R)$ and $\|A y\|>K$. Now (3.6) and Lemma 2.2 imply that

$$
\begin{aligned}
\|A y\| & \leq M_{1} \int_{0}^{1} H(s, s) h(s) f(y(s)) d s+\sum_{j=1}^{m} \Omega_{j}\left(y\left(t_{j}\right)\right) \\
& <M_{1}\left(\int_{0}^{1} H(s, s) h(s) f(y(s)) d s+\sum_{j=1}^{m} \Omega_{j}\left(y\left(t_{j}\right)\right)\right) .
\end{aligned}
$$

Fix $k \in\{0,1, \ldots, m\}$ and notice that (3.9), (3.12), (3.14) and Lemma 2.2 yield

$$
\begin{aligned}
\min _{t \in\left[a_{k}, b_{k}\right]} A y(t) & =\min _{t \in\left[a_{k}, b_{k}\right]}\left(\int_{0}^{1} H(t, s) h(s) f(y(s)) d s+\sum_{j=1}^{m} W_{j}(t, y)\right) \\
& \geq M_{2} \int_{0}^{1} H(s, s) h(s) f(y(s)) d s+c_{0} \sum_{j=1}^{m} \Omega_{j}\left(y\left(t_{j}\right)\right) \\
& \geq M\left(\int_{0}^{1} H(s, s) h(s) f(y(s)) d s+\sum_{j=1}^{m} \Omega_{j}\left(y\left(t_{j}\right)\right)\right) \\
& \geq \frac{M}{M_{1}}\|A y\|>\frac{M}{M_{1}} K \geq L .
\end{aligned}
$$

So we get for each $k \in\{0,1, \ldots, m\}$ that

$$
\psi(A y)=\min _{k \in\{0,1, \ldots, m\}} \min _{t \in\left[a_{k}, b_{k}\right]} A y(t)>L .
$$

Thus condition (H3) of Theorem 1.1 holds. By Theorem 1.1, $A$ has at least three fixed points, that is, (1.1) has at least three nonnegative solutions $y_{1}, y_{2}$ and $y_{3}$ such that

$$
\left\|y_{1}\right\|<r, \quad y_{2}(t)>L \quad \text { for } t \in\left[a_{k}, b_{k}\right], k \in\{0,1, \ldots, m\},
$$

and

$$
\left\|y_{3}\right\|>r \quad \text { with } \min _{k \in\{0, \ldots, m\}} \min _{t \in\left[a_{k}, b_{k}\right]} y_{3}(t)<L .
$$

The proof is complete.

We work through an example to illustrate our results.

EXAMPLE 3.1. Consider the following impulsive boundary value problem:

$$
\left\{\begin{array}{l}
y^{\prime \prime}(t)+\left[(y(t)-1)^{1 / 3}+1\right]=0, \quad t \in(0,1), t \neq \frac{1}{2}, \\
\Delta y\left(t_{1}\right)=\frac{1}{3} y\left(t_{1}^{-}\right), \quad t_{1}=\frac{1}{2} \\
\Delta y^{\prime}\left(t_{1}\right)=-\frac{2}{3} y\left(t_{1}^{-}\right), \quad t_{1}=\frac{1}{2} \\
y(0)=0, \quad y\left(\frac{2}{3}\right)=y(1)
\end{array}\right.
$$


where $h(t) \equiv 1, f(y)=(y-1)^{1 / 3}+1, \alpha=1, \eta=\frac{2}{3}$. It is easy to see that conditions (3.1)-(3.5) hold. Let $\Omega_{1}(u)=2 u / 3, c_{0}=\frac{1}{8}$; it follows that (3.6) and (3.9) hold. Since

$$
\sup _{t \in[0,1]} \int_{0}^{1} H(t, s) h(s) d s=\frac{21}{64}, \quad \min _{k \in 0,1} \min _{t \in\left[a_{k}, b_{k}\right]} \int_{a_{k}}^{b_{k}} H(t, s) h(s) d s=\frac{1}{32},
$$

taking $r=1, L=2$ and $R=91>L M^{-1} M_{1}=90$, then (3.7), (3.8) and (3.10) hold. So all the conditions of Theorem 3.1 hold. By Theorem 3.1, (3.15) has at least three nonnegative solutions.

\section{Acknowledgements}

This work is supported by the NNSF of China (No. 10571050 and No. 60671066), a project supported by the Scientific Research Fund of Hunan Provincial Education Department (07B041) and the Program for Young Excellent Talents in Hunan Normal University.

\section{References}

[1] R. P. Agarwal and D. O'Regan, "Multiple nonnegative solutions for second order impulsive differential equations", Appl. Math. Comput. 114 (2000) 51-59.

[2] R. P. Agarwal and D. O'Regan, "Existence of triple solutions to integral and discrete equations via the Leggett-Williams fixed point theorem", Rocky Mountain J. Math. 31 (2001) 23-25.

[3] R. P. Agarwal and D. O'Regan, "A multiplicity result for second order impulsive differential equations via the Leggett-Williams fixed point theorem", Appl. Math. Comput. 161 (2005) 433439.

[4] R. I. Avery and J. Henderson, "Three symmetric positive solutions for a second order boundary value problem", Appl. Math. Lett. 13 (2000) 1-7.

[5] R. W. Leggett and L. R. Williams, "Multiple positive fixed point of nonlinear operators on ordered Banach spaces", Indiana Math. J. 28 (1979) 673-688.

[6] R. Ma, "Positive solutions of a nonlinear three-point boundary value problem", Electron. $J$. Differential Equations 34 (1999) 1-8.

[7] J. Sun, W. Li and S. Cheng, "Three positive solutions for second-order Neumann boundary value problems", Appl. Math. Lett. 17 (2004) 1079-1084.

[8] P. J. Wang and R. P. Agarwal, "Criteria for multiple solutions of difference and partial difference equations subject to multipoint conjugate conditions", Nonlinear Anal. 40 (2000) 629-661.

[9] Q. Yao, "Successive iteration and positive solution for nonlinear second-order three-point boundary value problems", Comput. Math. Appl. 50 (2005) 433-444. 